\title{
INFLUENCE OF ELECTRON BEAM IRRADIATION ON PHYSICAL PROPERTIES OF MICROWAVE PLASMA SYNTHESIZED GRAPHENE NANOSHEETS
}

\author{
${ }^{1}$ Jana JURMANOVÁ, ${ }^{1}$ Ondřej JAŠEK, ${ }^{1}$ Jozef TOMAN, ${ }^{1}$ Miroslav ŠNÍRER, ${ }^{2}$ Michal KALINA \\ ${ }^{1}$ Department of Physical Electronics, Faculty of Science, Masaryk University, Brno, Czech Republic, EU, \\ janar@physics.muni.cz \\ 2Institute of Physical and Applied Chemistry, Faculty of Chemistry, Brno University of Technology, \\ Brno, Czech Republic, EU, kalina-m@fch.vut.cz
}

https://doi.org/10.37904/nanocon.2019.8453

\begin{abstract}
Scanning electron microscopy was used to determine physical properties of microwave plasma synthesized graphene nanosheets such as size and shape under various electron beam parameters (accelerating voltage, electron beam current, working distance). The stability and amount of defects of graphene nanosheets structure was investigated in dependence on these parameters and the results were compared to data obtained by TEM and Dynamic light scattering (DLS). Electron beam irradiation lead to change of nanosheets topography and defect formation was observed as well. The amorphization of the carbon nanostructure led to increase of intensity of $D$ band $\left(1360 \mathrm{~cm}^{-1}\right)$ in Raman spectra. Contrary to electron beam irradiation damage the high temperature annealing, up to $800^{\circ} \mathrm{C}$, in Ar led to no observable changes.
\end{abstract}

Keywords: Graphene, electron microscopy, irradiation, structure

\section{INTRODUCTION}

Carbon forms several allotropes with predominantly sp2 bond structure such as fullerenes [1], carbon nanotubes [2] and graphene [3]. These nanostructures can be prepared by wide range of methods including laser and arc discharge evaporation of carbon electrodes in He atmosphere [4] and plasma enhanced chemical vapour deposition using hydrocarbon or alcohol precursors in argon [5-6]. The stability of these structures is highly dependent on the amount of defects and overall sp2/sp3 bond ratio and strongly influences thermal stability and chemical reactivity of the material. Recently, high energy beam irradiation-induced damage to graphene has been investigated experimentally. The effects of plasma treatment, electron beam irradiation and low energy ions were studied in controlled way on graphene sheets to assess the influence of crystal structure and graphene properties with defects. Most studies deal with the low and medium energy electronbeam irradiation in the $\mathrm{eV}$ to $\mathrm{keV}$ energy regime [7-8]. In this energy regime the interaction regime of electrons and ions is dominated by elastic collisions, and the damage is due to nuclear collisions between impacting charged particles and carbon atoms [9].

Similarly, the choice of electron beam parameters can lead to enhanced contrast, but also damage the observed nanostructures deposited on dielectric substrate. Huang et al. [10] showed that by choosing appropriate acceleration voltage and working distance (WD) can lead to strong enhancement of image contrast and lower the noise level of imaged graphene sheets. This can be used not only to differentiate between substrate and graphene but also make distinction between graphene sheets with different thickness and features such as wrinkles and contaminants. These wrinkles and edges of carbon nanostructures can lead to field emission of electrons [11-12] and influence the quality of obtained image. Further decrease of acceleration voltage using cathode lens in STEM to tens of eVs [13-14] can be used to distinguish freestanding single layer graphene from thicker areas of the sample and topological defects. In this work we study the influence of 
electron beam parameters during scanning electron microscopy on contrast between graphene nanosheets and $\mathrm{SiO}_{2}$ substrate and on their properties.

\section{EXPERIMENTAL}

The graphene nanosheets were synthesized by ethanol decomposition in microwave plasma torch at atmospheric pressure. The microwave discharge was ignited inside reactor formed by quartz tube $(80 \mathrm{~mm}$ diameter, $200 \mathrm{~mm}$ length) terminated by dural flanges. The discharge electrode was hollow nozzle with central channel used for introduction of working gas - argon $(360-920 \mathrm{sccm})$ and subsequent ignition of plasma and secondary channel (annulus with outer radius $8.4 \mathrm{~mm}$ and inner radius $7.7 \mathrm{~mm}$ ) was used for introduction of carrying gas - argon (500 - $1400 \mathrm{sccm}$ ) with precursor (ethanol 2-25 sccm) vapours into the plasma environment. Synthesized nanopowder was collected from the reactor wall or on the $\mathrm{Si} / \mathrm{SiO}_{2}(92 \mathrm{~nm})$ substrates fixed in the holder Figure 1. More details about the experimental setup can be found in [15].

The thermal stability study was carried out by samples annealing in quartz glass tube $(100 \mathrm{~cm}$ long, inner diameter $45 \mathrm{~mm}$ ) placed in the centre of cylindrical horizontal furnace $(40 \mathrm{~cm}$ length, hot zone length of $15 \mathrm{~cm})$ terminated with flanges. The temperature inside the furnace was measured by the type $S$ thermocouple and controlled by electronic unit. The gas flow rates were controlled by electronic flow meters and the whole system was evacuated by a turbomolecular pump. The graphene nanosheets powder was put into a quartz boat and placed in the middle of the furnace. The annealing was done in the following sequence. The whole experimental setup was flushed by $\operatorname{Ar}$ flow $(2.5 \mathrm{lpm})$ for 5 minutes to avoid the air impurities during the annealing. The sample was heated to its target temperature $\left(350-800{ }^{\circ} \mathrm{C}, 25^{\circ} \mathrm{C} /\right.$ minute) under chosen atmosphere $\operatorname{Ar}(1000 \mathrm{sccm})$ or air and kept there for 15 minutes. The sample was then rapidly cooled by shifting the oven with regard to sample position by $30 \mathrm{~cm}$ and cooled down under Ar flow $1000 \mathrm{sccm}$ to the room temperature.

Raman spectroscopy was carried out using HORIBA LabRAM HR Evolution system with $532 \mathrm{~nm}$ laser, using $100 x$ objective and $25 \%$ ND filter in the range from 1000 to $3200 \mathrm{~cm}^{-1}$. Samples were imaged with TESCAN scanning electron microscope (SEM) MIRA3 with Schottky field emission electron gun equipped with secondary electron (SE) and back-scattered electron (BSE) detectors as well as characteristic X-ray detector Oxford Instruments EDX analyzer. Transmission electron microscopy was carried out using JEOL JEM-2100F microscope. Size distribution of prepared graphene nanosheets were determined also by means of dynamic light scattering using colloidal analyser Zetasizer Nano ZS (Malvern Pananalytical Ltd.).

\section{RESULTS AND DISCUSSION}

\subsection{Determination of graphene nanosheets size}

Electron microscopy was used as primary technique to determine the size of graphene nanosheets. In general the nanosheet shape was rectangular but their edges are often curled and rolled up which led to their deformation and difficulties in determination of exact size. Therefore, least deformed and clearly visible, not aggregated, nanosheets were selected for image analysis. The size of nanosheets determined from SEM images had length $(176 \pm 11) \mathrm{nm}$ and width $(101 \pm 16) \mathrm{nm}$ - see Figure 2. This is in agreement with size determined from TEM micrographs Figure 3 (length $(178 \pm 16) \mathrm{nm}$ and width $(128 \pm 6) \mathrm{nm}$. To compare the obtained value with independent technique, the samples were analyzed by DLS. The obtained distribution of particle sizes can be seen in Figure 4. The size distribution function is skewed to the larger values with the average particle size $(459 \pm 6) \mathrm{nm}$. The particle size uncertainty refers to three independent measurements of average particle size. This significant shift can be attributed to the formation of dimers or trimers in dispersion and presence of solvation layer around the analysed graphene nanosheets, which overestimates the measured size in DLS measurement in comparison to SEM and TEM. 


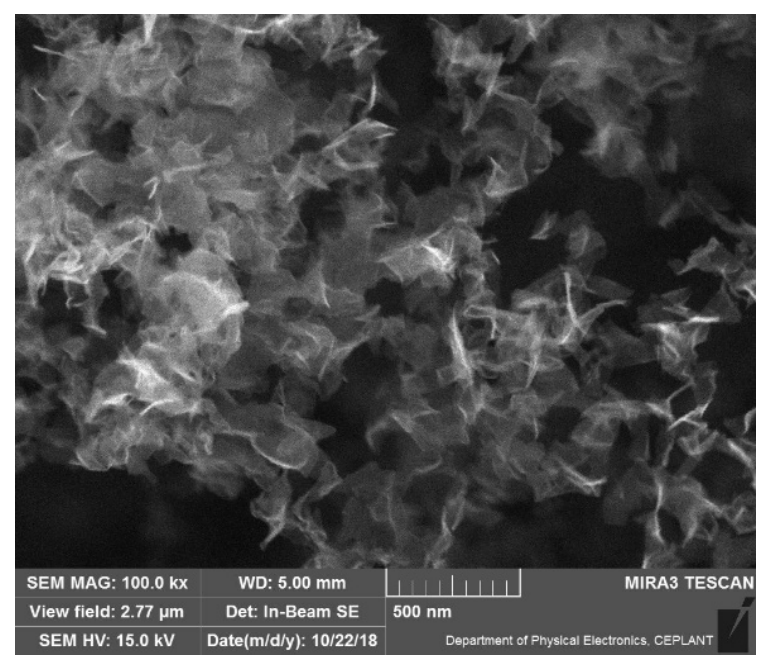

Figure 1 SEM image of as deposited nanosheets

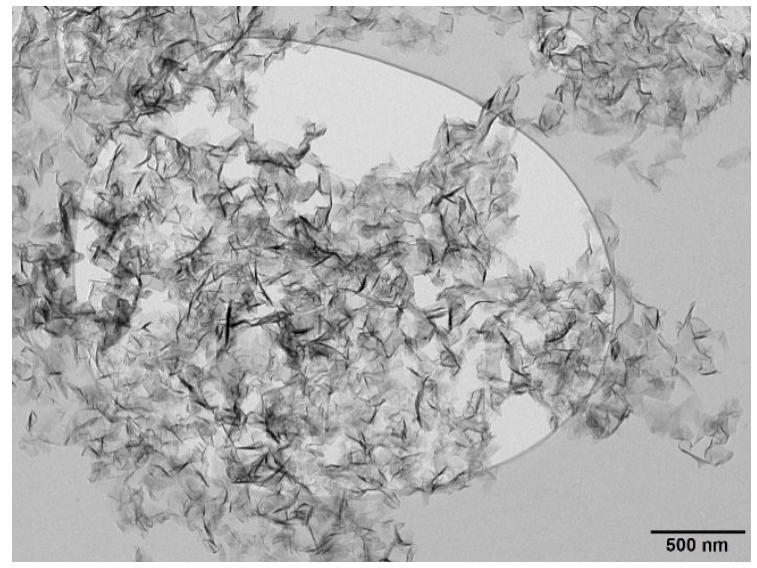

Figure 3 TEM image of graphene nanosheets

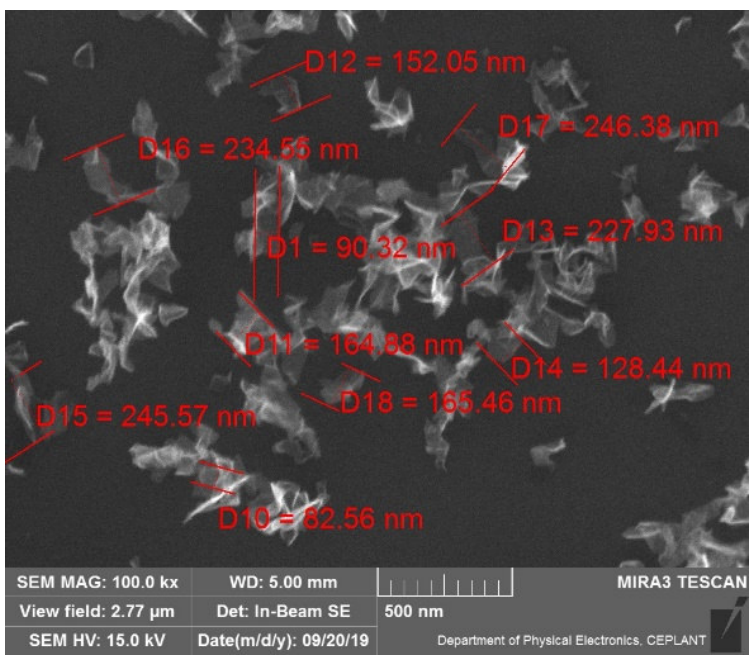

Figure 2 Determination of nanosheets size by SEM

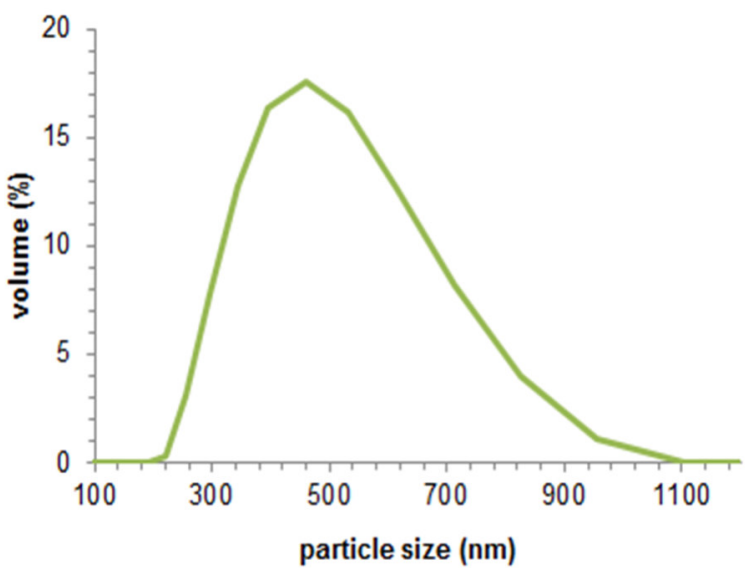

Figure 4 DLS size distribution of graphene nanosheets

\subsection{Enhancement of imaging contrast of graphene nanosheets on $\mathrm{SiO}_{2}$ substrate}

To improve imaging and determination of areas of overlapping nanosheets and curled edges we investigated the influence of working parameters on image contrast. In comparison with Huyang, we tried to change the image parameters to obtain the best contrast for graphene nanosheets. The nanosheets are small, hundreds of $\mathrm{nm}$, so we need to get an image with the best resolution. This requirement leads to the choice of higher values of acceleration voltage and short working distance. However, as Huang has shown, it is far preferable to choose a rather long working distance to obtain a high contrast between the substrate $\left(\mathrm{SiO}_{2}\right)$ and graphene. Better contrast values (CV), similarly to work of Huang et al. [10] were based on longer working distances. The reason was minimization of the contribution of electrons of type SE3 (originate form backscatter electron colliding with chamber walls and other interior components). Because the escape area of these electron is large (tens to hundreds of nanometers), the resolution increases as the number of these electrons decreases. Contrary to Huang our contrast was negative, we obtained the light graphene on the dark silicon dioxide. The reason could be due to a poorer conductive connection of the individual graphene nanosheets to the substrate, which increased nanosheets charging. In dependence on WD and acceleration voltage we investigated CV between carbon nanosheets and Figure 5. 

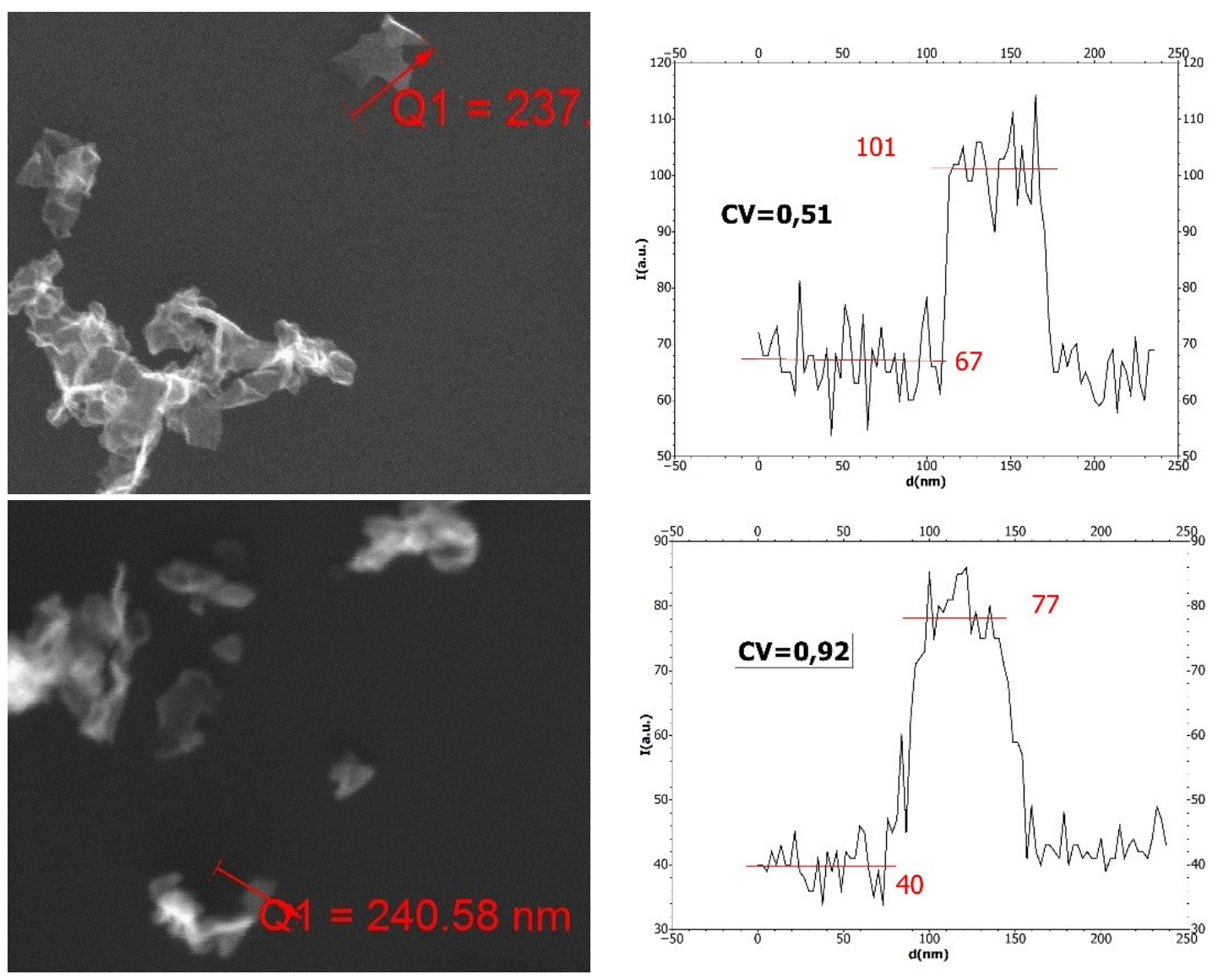

Figure 5 The comparison of the resolution and contrast (CV) for working distance $5 \mathrm{~mm}$ (above) and $40 \mathrm{~mm}$ (below), acceleration voltage of $20 \mathrm{kV}$

In this way, we could use enhanced CV to determine boundaries between two overlapping sheets and their edges - Figure 6.
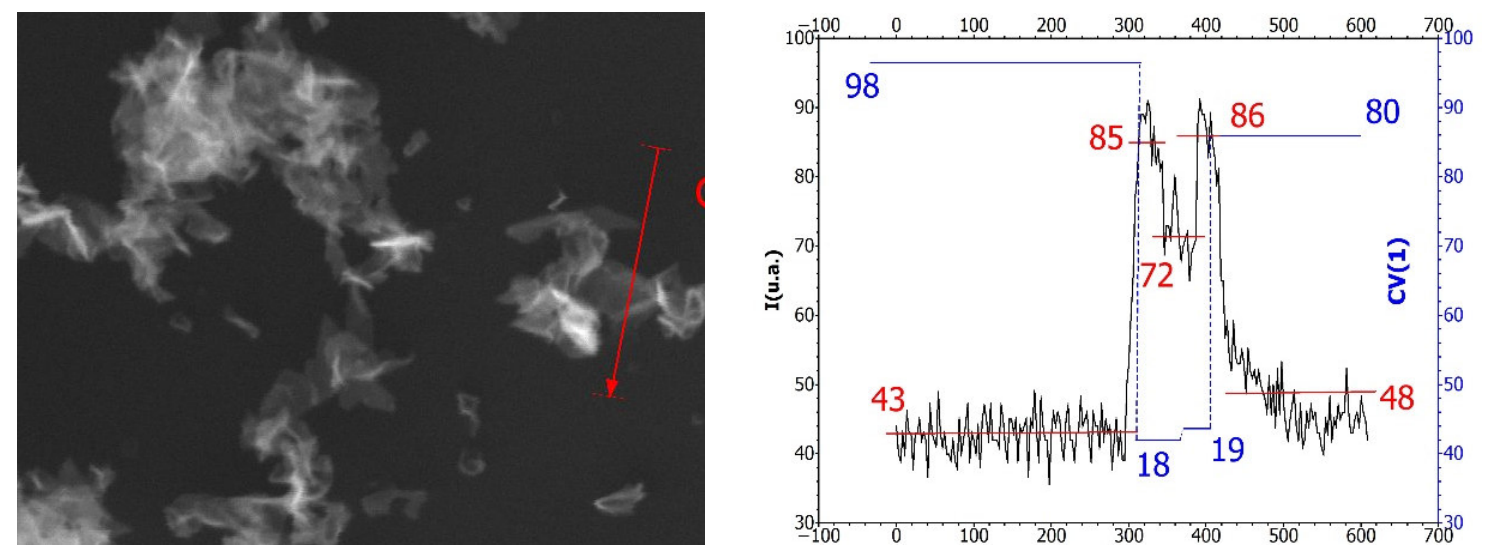

Figure 6 Enhanced contract in SEM between two graphene nanosheets and their edges WD 20mm, acceleration voltage $20 \mathrm{kV}$

\subsection{Influence of electron beam irradiation on nanosheet imaging}

To investigate the influence of the electron beam on the stability of graphene nanosheets, we applied different electron beam current, acceleration voltage, and different exposure time for nanosheet analysis. The beam 
current was varied from $90 \mathrm{pA}$ to $100 \mathrm{pA}$, acceleration voltage from $5 \mathrm{kV}$ to $30 \mathrm{kV}$ to reach varying dose (energy per area). The acquisition time was $32 \mathrm{~s}$, so the electron beam stays 35 microseconds on one pixel $(2.7 \mathrm{x}$ $2.7 \mathrm{~nm}$ ) and the pixel obtains the dose about 50 pikoJ. The first total dose absorbed by the imaged nanosheet was 8.5 microjoule. The second absorbed dose was 30.7 microjoule Figure 7 . The highest dose led to amorphization of the structure which was also observed in Raman spectra by increase of $D$ band $\left(1360 \mathrm{~cm}^{-1}\right)$ intensity.

These results are consistent with the work of Teweldebrhan et al. [7] which showed that graphene lattice is strongly influenced by electron beam of energy between $5-20 \mathrm{kV}$ and leads to strong enhancement of $D$ band $\left(1345 \mathrm{~cm}^{-1}\right)$ in Raman spectra and leads to amorphization of carbon material. While this process results in graphene damage it can be used to create various features such as nanopores, slits and gaps in graphene sheets as shown by Fischbein et al. [8].
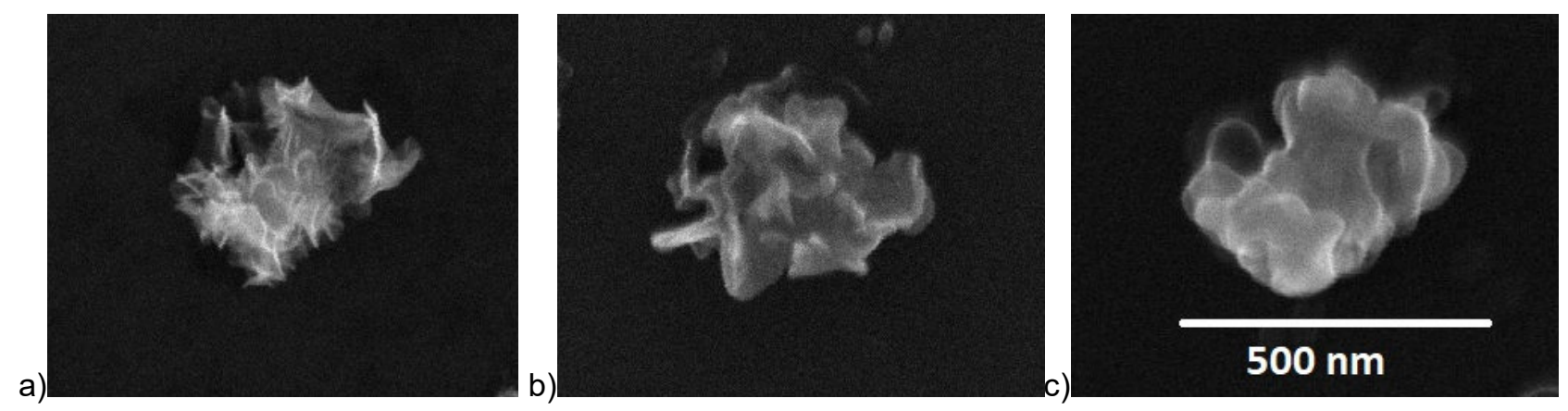

Figure 7 The same nanosheet a) as deposited b) after $8.5 \mu \mathrm{J}$ dose and c) after next $30.7 \mu \mathrm{J}$ dose

\subsection{Influence of high temperature annealing on nanosheet structure}

High temperature annealing in vacuum, $\mathrm{Ar}$ and oxygen containing atmosphere was carried out in temperate range of 350 to $800{ }^{\circ} \mathrm{C}$. In the case of vacuum and Ar atmosphere annealing, no significant changes were observed in the structure of the annealed material in whole temperature range as observed by SEM and as shown by Raman spectroscopy analysis. Intensity and ratio of $D\left(1360 \mathrm{~cm}^{-1}\right), G\left(1580 \mathrm{~cm}^{-1}\right)$ and second order $2 \mathrm{D}\left(2690 \mathrm{~cm}^{-1}\right)$ bands, $\mathrm{I}_{\mathrm{D}} / \mathrm{I}_{\mathrm{G}}$ of 0.5 and $\mathrm{I}_{2 \mathrm{D}} / \mathrm{I}_{\mathrm{G}}$ ratio of 0.6 remain the same up to $700{ }^{\circ} \mathrm{C}$ and $\mathrm{ID}_{\mathrm{D}} / \mathrm{I}_{\mathrm{G}}$ ratio decreased and $\mathrm{I}_{2 \mathrm{D}} / \mathrm{l}_{\mathrm{G}}$ ratio increased at $800{ }^{\circ} \mathrm{C}$ suggesting removal of defects in the structure. In the case of oxygen containing atmosphere the trend was opposite and above $650^{\circ} \mathrm{C}$ the material started to oxidize and was burnt out above $700{ }^{\circ} \mathrm{C}$. The increasing $\mathrm{l} / \mathrm{l} / \mathrm{G}$ ratio could be understood in terms of increasing number of defects due to the reaction of carbon atoms with oxygen and further disintegration of graphene nanosheet.

\section{CONCLUSIONS}

Influence of electron beam parameters in scanning electron microscope on imaging and properties of graphene nanosheets was investigated. The size of nanosheets determined from SEM was slightly smaller than size determined from TEM analysis. This result was partially caused by fuzzy image contrast of SEM especially in case of nanosheets edges. Therefore, we optimized our imaging setting to enhance contrast between substrate and nanosheets. Deformation of nanosheets was investigated in dependence on electron beam irradiation and high temperature annealing. While high temperature annealing in vacuum and argon atmosphere had no observable effect on nanosheets size and structures, as determined by SEM and Raman spectroscopy, the high intensity electron beam irradiation, above a few microjoule per nanosheet, led to modification of graphene edges and change of shape of observed nanostructures. 


\section{ACKNOWLEDGEMENTS}

This work was supported by The Czech Science Foundation (GA CR) under project 18-08520S and in part by the project LO1411 (NPU I) funded by Ministry of Education, Youth and Sports of Czech Republic. We would like to thank Jiri Bursik for TEM analysis of graphene nanosheets.

\section{REFERENCES}

[1] H. W. KROTO, J. R. HEATH, S. C. O'BRIEN, R. F. CURL, and R. E. SMALLEY, "C60: Buckminsterfullerene," Nature, 1985, vol. 318, no. 6042. pp. 162-163.

[2] S. IIJIMA, "Helical microtubules of graphitic carbon," Nature, 1991, vol. 354, no. 6348. pp. 56-58.

[3] K. S. Novoselov et al., "Two-dimensional atomic crystals," Proc. Natl. Acad. Sci. U. S. A., 2005, vol. 102, no. 30, pp. 10451-10453.

[4] A. C. FERRARI et al., "Science and technology roadmap for graphene, related two-dimensional crystals, and hybrid systems," Nanoscale, 2015, vol. 7, no. 11, pp. 4598-4810.

[5] A. DATO, "Graphene synthesized in atmospheric plasmas-A review," Journal of Materials Research, 2019 , vol. 34, no. 1. pp. 214-230.

[6] M. ELIÁŠ et al., "Atmospheric pressure barrier discharge at high temperature: Diagnostics and carbon nanotubes deposition," Journal of Applied Physics, 2015, vol. 117, no. 10. p. 103301.

[7] D. TEWELDEBRHAN and A. A. BALANDIN, "Modification of graphene properties due to electron-beam irradiation," Applied Physics Letters, 2009, vol. 94, no. 1. p. 013101.

[8] M. D. FISCHBEIN and M. DRNDIĆ, "Electron beam nanosculpting of suspended graphene sheets," Applied Physics Letters, 2008, ol. 93, no. 11. p. 113107.

[9] J. ZENG et al., "Irradiation effects of graphene and thin layer graphite induced by swift heavy ions," Chinese Physics B, 2015, vol. 24, no. 8. p. 086103.

[10] L. HUANG, D. ZHANG, F.-H. ZHANG, Z.-H. FENG, Y.-D. HUANG, and Y. GAN, "High-Contrast SEM Imaging of Supported Few-Layer Graphene for Differentiating Distinct Layers and Resolving Fine Features: There is Plenty of Room at the Bottom," Small, 2018, vol. 14, no. 22, p. e1704190.

[11] J. PEKAREK et al., "MEMS Carbon Nanotubes Field Emission Pressure Sensor With Simplified Design: Performance and Field Emission Properties Study," IEEE Sensors Journal, 2015, vol. 15, no. 3. pp. 1430-1436.

[12] A. KNÁPEK, D. SOBOLA, P. TOMÁNEK, Z. POKORNÁ, and M. URBÁNEK, "Field emission from the surface of highly ordered pyrolytic graphite," Applied Surface Science, 2017, vol. 395. pp. 157-161.

[13] E. MIKMEKOVÁ et al., "Very low energy electron microscopy of graphene flakes," J. Microsc., 2013, vol. 251, no. 2, pp. 123-127.

[14] K. L. MAN and M. S. ALTMAN, "Low energy electron microscopy and photoemission electron microscopy investigation of graphene," Journal of Physics: Condensed Matter, 2012, vol. 24, no. 31. p. 314209.

[15] J. TOMAN, O. JASEK, M. SNIRER, V. KUDRLE, and J. JURMANOVA, "On the interplay between plasma discharge instability and formation of free-standing graphene nanosheets in a dual-channel microwave plasma torch at atmospheric pressure," Journal of Physics D: Applied Physics, 2019, vol. 52, no. 26. p. 265205. 